\title{
Own related work
}

Several parts of this monograph are related to published studies: ${ }^{14}$ most of them in peer-reviewed journal papers, some as peer-reviewed conference papers, some nonreviewed work (GAMM Leitartikel or lecture notes), and some under current review. My own contributions to phase-field fracture, multiphysics extensions, and collaborations ${ }^{15}$ can be roughly divided into the following categories:

1. Basic concepts in phase-field fracture numerical modeling:

(a) Crack irreversibility constraint with simple penalization [323], augmented Lagrangian [430], primal-dual active methods [221], and complementarity systems [301]

(b) Higher-order finite elements [301, 249]

(c) Mixed formulations for (nearly) incompressible fracture [301, 42]

(d) Monolithic [444, 445], quasi-monolithic [221, 444], and iterative coupling schemes $[88,154,247]$ of the displacement/phase-field system

(e) Nonlinear monolithic solvers [444, 445]

(f) Parallel multigrid preconditioners [222] (AMG) and [250, 249] (matrix-free GMG)

2. Adaptive modeling, error control, and local mesh refinement:

(a) Predictor-corrector scheme for mesh refinement around the crack [221] (2d), $[448,285](3 \mathrm{~d})$

(b) Residual-based error estimators [299, 42]

(c) Goal-oriented error estimators [442]

(d) Multiple goal functionals for the perfect slit (not yet phase-field) [153]

(e) Adaptive nonintrusive global-local approach [341, 9]

(f) Adaptive time/loading step control $[443,159]$

3. Numerical optimization with phase-field fracture ${ }^{16}$

(a) Optimal control [336, 337]

(b) Parameter estimation [259, 342]

4. Engineering applications:

(a) Fracture and damage in screws [433]

(b) Fracture and damage in nearly incompressible rubber-like solids [298, 301]

(c) One-way coupling to a reservoir simulator in porous media [450]

(d) Fracture initialization with probability maps [287]

(e) Uniaxial compression test with inclined notch [160]

14 Complete publication lists on http://www.thomaswick.org/

15 All of these collaborations are highly appreciated and they are influential in this monograph.

16 Numerical optimization will not be touched in this monograph. For details, I refer immediately to the four listed papers/preprints [336, 337, 259, 342] and in multiphysics fluid-structure interaction to [378, 452] and recent differentiability results for stationary problems [451]. 
5. Multiphysics modeling and applications:

(a) Mathematical and numerical modeling of pressurized fractures in porous media [324, 323, 448, 285, 9]

(b) Mathematical and numerical modeling of fluid-filled fractures in porous media [321, 322, 285, 286, 14]

(c) Nonlinear porous media phase-field fracture [425]

(d) Transport flow in phase-field fractures in porous media [286]

(e) Two-phase flow in phase-field fractures in porous media [280]

(f) Nonisothermal modeling of pressurized fractures in porous media [343]

(g) Coupling to fluid-structure interaction [441, 443]

(h) IPACS: Integrated Phase-Field Advanced Crack Propagation Simulator. An adaptive, parallel, physics-based-discretization phase-field framework for fracture propagation in porous media [431]

6. Benchmarking:

$[449,222]$

7. Research software development:

[222, 431, 207, 183]

8. GAMM Leitartikel:

[446]

9. Lecture notes as educational advancements:

(a) Phase-field fracture lecture notes [300] [partially taken and re-worked in this monograph]

(b) $[447$, specifically Chapters 9, 11-14] [few materials taken in this monograph] 